\title{
La situation choquante de la femme dans deux romans francophones de Tahar Ben Jelloun et Mariama Bâ : L'Enfant de Sable et Une si longue lettre
}

\author{
par \\ Mohamed Abdel Tawab Abdel Latif* \\ Mohamedabdeltawab_256@yahoo.com
}

\section{Résumés}

La situation choquante de la femme dans deux romans francophones chez (Tahar Ben Jelloun et Mariama Bâ

Nous allons tenter dans notre recherche d'analyser comment la femme est représentée dans l'œuvre romanesque de Tahar Ben Jelloun de Mariama Bâ. Les deux romanciers actualisent, à travers leurs œuvres romanesques l'enfant de sable et Une si longue lettre un sujet très épineux et très brûlant dans la société marocaine qu'est la condition de la femme. Le sixième roman de Tahar Ben Jelloun entretient une bonne réflexion sur le statut de la femme au Maroc à partir des années cinquante jusqu'à l'obtention de ses droits sociaux et judicaires au tournant de l'an deux mille et celle aussi de Mariama Bâ qui aborde le même sujet mais sous un autre angle : la polygamie.

Mots- clés:Le féminisme - la société patriarcale- Tahar Ben Jelloun- Mariama Bâ

* maitres de conférences à la faculté des Lettres-Université du Fayoum

(La situation choquante de la femme...) Dr. Mohamed Abdel Latif 


\section{- Introduction de la recherche}

La littérature francophone fait partie intégrante de la littérature française. C'est un terrain fertile où se mêlent toutes les questions qui préoccupent le monde francophone; elle est considérée donc comme le fruit de la colonisation.

Les écrivains francophones ont opté pour la langue française de l'ancien colonisateur comme langue d'écriture afin d'aborder les thèmes qui touchent leurs propres sociétés et leurs pays respectifs; tels, les thèmes de la guerre, de la colonisation, de l'identité et de l'immigration. Ces écrivains ont tous abordé un thème très délicat voire angoissant et qui mérite d'être discuté au sein de la littérature francophone d'expression française à savoir celui de la situation choquante de la femme dans leurs milieux. Cette étude se propose donc d'analyser ce sujet à travers L'Enfant de Sable de Tahar Benjelloun et Une si longue lettre de Mariama Bâ.

Depuis l'aube de l'histoire, la femme a été estimée comme une créature faible, une honte à s'en

(La situation choquante de la femme...) Dr. Mohamed Abdel Latif 
débarrasser. Selon les préjugés masculins, elle est créée justement dans la vie pour s'occuper de ses enfants et rester attachée à son domicile. Elle n'a pas le droit de s'exprimer même en ce qui concerne sa vie privée. Dès lors, elle est exclue de toute communication avec le milieu social.

A titre d'exemple, pendant la colonisation française au Maroc, les femmes préféraient l'isolement, l'enfermement et l'envie de ne pas sortir de chez elles, de peur d'être agressées par l'excolonial. Tahar Ben Jelloun et Mariama Bâ ont utilisé une langue ayant un statut international, comme le montre cet extrait «le français est une langue qui est porteuse d'un message de liberté et d'égalité héritée de la Révolution » (Combe, 1995, p. 16), afin de donner l'occasion aux lecteurs des quatre coins du monde de découvrir le long chemin parcouru par la femme en vue d'obtenir ses droits bafoués surtout dans une société patriarcale où l'homme occupe une place très prépondérante.

(La situation choquante de la femme...) Dr. Mohamed Abdel Latif 


\section{- Problématique de la recherche}

Le sixième roman de Tahar Ben Jelloun entretient une bonne réflexion sur le statut de la femme au Maroc à partir des années cinquante jusqu'à l'obtention de ses droits sociaux et judicaires au tournant de l'an deux mille ; celui de Mariama Bâ aborde le même sujet mais sous un autre angle : la polygamie.

Ce qui est intéressant dans ces deux ouvrages c'est qu'ils sont écrits par deux personnes de sexe différent, deux pensées différentes, l'une représente la nature féminine et l'autre symbolise l'agent masculin. Les deux romans sont écrits également avec un vocabulaire très varié.

La question qui se pose maintenant : Comment les deux écrivains ont présenté la femme dans leurs œuvres romanesques et comment elle a évolué entre les deux romans ? Est-ce qu'ils la représentent objectivement ou plutôt selon leur nature biologique surtout dans une telle société africaine où les hommes dominent?

(La situation choquante de la femme...) Dr. Mohamed Abdel Latif 
Notre choix de l'écrivaine sénégalaise Mariama Bâ a pour but de montrer un aspect très intéressant de ses écrits : l'engagement féministe.

Dans cette recherche, nous analysons l'image de la femme, à travers les personnages principaux de chaque écrivain, et leur vision qui concerne les relations hommes-femmes au sein de la société africaine.

Nous discutons aussi le thème de la marginalisation de la femme et le rejet de ses droits personnels, sociaux et judiciaires au sein d'une société patriarcale en Afrique où l'homme domine.

\section{- Motif du choix}

Une fois le joug du colonialisme français ébranlé, notamment dans les années cinquante, une question s'imposait dans les écrits francophones sur les conditions d'existence de la femme sous la forme d'une autofiction romanesque. La majorité des lecteurs francophones critique le fait de passer sous silence le rôle majeur de la femme dans la société, l'importance qu'elle occupe au sein de la famille et

(La situation choquante de la femme...) Dr. Mohamed Abdel Latif 
sa lutte incessante en faveur de l'égalité des droits entre l'homme et la femme (....) (Rey, 2006).

$\mathrm{Au}$ fil du temps les orientations des romanciers francophones vers la cause de la femme ont changé selon l'époque et la société notamment à partir des années quatre-vingts où les écrits au féminin changent d'orientation en se mettant à aborder des thèmes épineux tels la marginalisation et la maltraitance de la femme au sein de la société africaine à cause des traditions et du colonialisme (...) (Assad, 2012, p. 34), ou encore la polygamie. Parmi les écrivains francophones qui ont manipulé leurs œuvres romanesques pour revendiquer un changement social Tahar Benjelloun et Mariama Bâ. Ils se sont efforcés de rénover la vie de la femme du milieu africain francophone en vue de la revaloriser et la rehausser.

(La situation choquante de la femme...) Dr. Mohamed Abdel Latif 
- Le corpus

1-Tahar Ben Jelloun, L'Enfant de sable, Éditions du Seuil, Paris, 1985.

2- Mariama Bâ : Une si longue lettre, Nouvelles Éditions Africaines, Paris, 1979

\section{- Synopsis des deux romans}

\section{A- L'Enfant de sable}

Ce roman est inspiré d'un fait divers authentique. Il raconte la vie d'Ahmed, huitième fille d'une famille qui, sans héritier mâle, décide d'élever cette fille au prénom masculin comme un garçon, sans prendre en considération le trouble et l'incertitude qui résulteraient de cette décision. À son tour, Ahmed choisit d'assumer la révolte de son père, de vivre en homme et d'épouser une fille abandonnée qui participera bientôt à une véritable descente aux enfers due à un mensonge social des plus absurdes.

\section{B- Une si longue lettre}

Ramatoulaye est l'héroïne de ce roman. Elle raconte l'histoire d'une manière épistolaire. Elle écrit

(La situation choquante de la femme...) Dr. Mohamed Abdel Latif 
une "si longue une longue lettre" à Assaïtou, sa meilleure amie, la destinataire. Une si longue lettre aborde la vie quotidienne des femmes mais surtout la douleur de l'héroïne quand son mari prend une seconde épouse après vingt-cinq ans de mariage et de fréquentation. Les deux amies vivent au Sénégal et sont toutes deux confrontées au même problème, celui de l'arrivée imposée d'une coépouse. Cependant, elles se comportent différemment. D'ailleurs, Tante Nabou et Farmata sont deux autres femmes qui jouent un rôle secondaire dans ce roman. La première est la belle-mère d'Assaïtou et la seconde est la voisine de Ramatoulaye.

Nous arrivons à l'analyse de la conception de la marginalisation de la femme dans le roman francophone à la lumière du corpus. Nous allons voir aussi comment les deux écrivains ont eu recours aux procédés d'écriture romanesques pour bien analyser l'état psychologique et la vie quotidienne de chaque personnage afin de permettre aux lecteurs de faire un rapport entre son état psychique et sa vie personnelle au sein de sa famille.

(La situation choquante de la femme...) Dr. Mohamed Abdel Latif 
Ce travail est censé être une concrétisation du grand trajet parcouru par la femme africaine francophone depuis son indépendance jusqu'à nos jours.

\section{I- La marginalisation de la femme au}

\section{sein de la société africaine}

Selon Assia Djebar «toute femme s'appelle blessure » (Mortimer, 1988, p. 8). Cette forme métaphorique se voit comme une maxime et résonne dans les esprits, lorsqu'on tient compte des constituants de sa syntaxe. Cette forme figurative, qui émane d'un raisonnement logique, s'adapte à la femme africaine francophone et tout particulièrement à la femme marocaine dont L'Enfant de sable est un modèle. Selon la pensée du romancier, toutes les femmes, nées au Maroc, sont emprisonnées dans des espaces étroits où elles sont isolées et barricadées dans des rôles secondaires et dépendants. Elles n'ont ni valeur ni rôles à jouer car ceux-ci sont quasiment identiques.

De plus, les charges sociales, qui leur incombent rendent leur vie personnelle et leur quotidienneté

(La situation choquante de la femme...) Dr. Mohamed Abdel Latif 
infernales et font d'elles des victimes soumises au patriarcat de l'homme.

Dans L'Enfant de sable, nous trouvons que Benjelloun interprète la souffrance de la femme d'après sa relation avec l'homme. Elle est toujours victime d'un grand manque d'affection, d'une indifférence inhumaine et violente et d'un patriarcat brutal au sein duquel la femme ne connaît que dépendance et soumission. C'est l'image reflétée par le roman, les sept filles de Hadj Ahmed incarnent cette souffrance. L'amour paternel dont elles devaient jouir, elles en sont profondément privées :

"Comme il ne pouvait pas s'en débarrasser, il cultivait à leur égard non pas de la haine, mais de l'indifférence. Il vivait à la maison comme s'il n'avait pas de progéniture. Il faisait tout pour les chasser de sa vue" (Jelloun, 1985, p. 18).

Le père de famille se chevauche en ce qui concerne les valeurs de la domination de l'homme au sein du foyer conjugal. Il réserve uniquement à ses enfants filles une place inférieure qui ne s'adapte

(La situation choquante de la femme...) Dr. Mohamed Abdel Latif 
pas du tout à la position dont jouit le père géniteur vis-à-vis de ses enfants au milieu de sa famille. Par conséquent, les filles sont repoussées et rejetées par lui. De surcroit, il a fait d'elles des membres infirmes et de véritables orphelines. La déception dont souffre le personnage du père provient de son orgueil blessé ; à savoir du fait qu'il désespère de ne pas avoir un mâle héritier qui porte le nom de la famille et qui peut finalement l'immortaliser.

Dans le même ordre d'idées, le mâle devrait accorder à son père une place très distinguée au sein de la communauté à laquelle le père appartient ; surtout au moment où ses frères attendent impatiemment qu'il meure pour s'emparer de ses biens. Cependant, la fille est considérée comme une personne qui vit négligée et inaperçue sous le toit paternel, ce qui mène à une grande exclusion au sein de sa demeure.

De plus, les préjugés socioculturels l'empêchent d'être tendre ; au contraire, il est ingrat et haineux vis- à-vis de ses soeurs. Le père ne s'en cache point, comme le prouve cet extrait :

(La situation choquante de la femme...) Dr. Mohamed Abdel Latif 
«Bien sûr tu peux me

reprocher de ne pas être tendre avec tes filles. Elles sont à toi. Je leur ai donné mon nom. Je ne peux pas leur donner mon affection parce que je ne les ai jamais désirées. (...) (Jelloun, 1985, p. 19).

Le discours du père met en relief l'ampleur de l'angoisse existentielle dont souffre la femme dans les pays musulmans. D'autre part, l'auteur, pour confirmer ce drame et attirer l'attention du lecteur, emploie le pronom personnel 'toi, tes'. Cet emploi illustre le rapport de distanciation père/filles grâce au concept de jonction et de disjonction analysé par Assaraf (ASSARAF, 1993). Il dit que l'incommunicabilité verbale entre père/filles annule tout lien de cohésion et d'entente entre eux au sein de la même communauté. Il incarne l'état d'indifférence profonde de cet homme à l'égard ses filles. Le père leur parle comme si elles étaient des inconnues ou des étrangères qui habitent son domicile.

(La situation choquante de la femme...) Dr. Mohamed Abdel Latif 
De surcroît, Il n'a jamais dit un mot susceptible de réveiller ou de révéler son amour paternel. La preuve en est dans ces propos:

$$
\text { "Il ne se souvenait pas }
$$

d'avoir posé sa main un jour sur le visage de l'une de ses filles. Entre lui et elles il avait élevé une muraille épaisse» (Jelloun, 1985, p. 22).

La muraille est ici le symbole de l'incommunicabilité et le non contact entre le père et ses filles. Elle est munie d'un écho dramatique dans la mesure où elle matérialise la dépravation affective des filles et anéantit complètement leur présence. L'indifférence de cet homme interprète tout ce que nous venons d'expliquer au-dessus. La nonchalance s'éclaircit aussi dans la manière dont on présente la femme dans une société soumise aux traditions ancestrales où la loi dominant est celle du mâle.

En outre, cette indifférence donne l'occasion au lecteur d'imaginer l'excès de violence qui transforme le père en un vrai sadique.

(La situation choquante de la femme...) Dr. Mohamed Abdel Latif 
D'autre part, lorsque la mère est emmenée au Hammam chaque semaine par Ahmed, elle nous décrit la libération des femmes des violences qu'elles subissent des hommes au quotidien. Il nous appartient maintenant de jeter la lumière sur les formes de violence masculine au sein de la famille : d'après la première lecture de L'Enfant de sable, nous constatons que le personnage masculin est un représentant de la force, de la virilité mais en parallèle de la dégradation. Ainsi, la femme souffretelle sous son autorité jusqu'à la mort. La violence va plus loin pour dépasser les formes traditionnelles que tout le monde connaît: elle est d'abord psychique, à partir du moment où l'épouse est blessée dans ses instincts, ses sens et ses besoins. La femme n'a pas le droit de se libérer ni de son défoulement ni de ses impulsions. Si elle le déclare, elle serait impolie et issue d'une mauvaise famille ; elle ne s'en libère que pendant le Hammam où toutes les femmes se trouvent seules loin du monde extérieur des hommes, comme le dit Andrea : «le Hammam est le lieu de bonheur et de divertissement pour les femmes marocaines » (Andréa, N013 2007).

(La situation choquante de la femme...) Dr. Mohamed Abdel Latif 
De plus, la violence physique est également ancrée dans le châtiment. Voici un extrait qui met en lumière ce sujet : «Il avait même amené sa femme à séjourner dans un marabout durant sept jours et sept nuits, se nourrissant de pain sec et d'eau. Elle s'était aspergée d'urine de chamelle $»(J e l l o u n, 1985$, p. 17). Selon la conception du narrateur : le corps féminin n’appartient pas à la femme mais plutôt à son mari. La punition à laquelle est soumise la femme interprète les fardeaux que le père inflige à ses enfants-filles. Il y exerce des droits qui selon le père sont enracinés et figés. Il obéit toujours à ses inclinations masculines même si elles sont contre les ordres de Dieu comme le prouve cette citation :

$$
\begin{aligned}
& \text { «Les sévices infligés à } \\
& \text { cette "véritable usine à } \\
& \text { fabriquer des filles "obéissent } \\
& \text { à la logique bien masculine } \\
& \text { d'un père en colère contre } \\
& \text { l'arbitraire divin, et déterminé } \\
& \text { par-dessus tout à en découdre } \\
& \text { avec la fatalité » (Jelloun, } \\
& \text { 1985, p. 22). }
\end{aligned}
$$

(La situation choquante de la femme...) Dr. Mohamed Abdel Latif 
Le père ne justifie jamais les actes atroces et inhumains envers ses filles ou sa femme, c'est le travail à la massification.

Dans cette perspective, elles n'ont qu'à endurer jusqu'au bout : "Elle avait bu un liquide saumâtre et très amer préparé par une vieille sorcière. Elle eut de la fièvre, des nausées insupportables, des maux de tête. Son corps s'usait. Son visage se ridait. Elle maigrissait et perdait souvent conscience »(Jelloun, 1985, p. 21).

Le père n'a jamais cessé de commettre de tels actes envers ces êtres faibles. Il ressemble à un bourreau, à un berger qui dirige des bêtes de somme profitant ainsi de sa nature biologique. La torture qu'elles subissent interprète bien les pratiques bien africaines comme l'excision et l'infibulation ou bien le repassage des seins, comme le mentionnent les personnages féminins créées par la romancière Mariama Bâ :

«La femme, dans cette cuvre, habite un corps brûlé et noirci par toutes les formes d'oppression imposées par la société patriarcale et l'idéologie phallocentrique» (Béatrice, 1997, p. 39)

(La situation choquante de la femme...) Dr. Mohamed Abdel Latif 
Pour accabler la féminité de la fille et réaliser le dessein de son mari, la femme va torturer le corps de sa fille en vue d'effacer tout signe de féminité chez elle :

«Elle s'inquiétait pour ma poitrine qu'elle pansait avec du lin blanc; elle serrait très fort les bandes de tissu fin au risque de ne plus pouvoir respirer. Il fallait absolument empêcher l'apparition des seins. La bande de tissu autour de la poitrine me serrait toujours» (Jelloun, 1985, p. 38).

D'une autre manière, l'idée de la marginalisation chez Mariama Bâ prend une autre forme : Elle critique la culture musulmane qui donne le droit à l'homme de la société sénégalaise d'être polygame sans prendre la permission de sa première épouse.

Nous allons tenter d'analyser cette forme d'infidélité conjugale du mari envers son épouse d'après Ramatoulaye, l'héroïne d'une Si longue lettre.

(La situation choquante de la femme...) Dr. Mohamed Abdel Latif 
Au début, 1'époux de Ramatoulaye Modou Fall paraît comme un homme dominant et décidant dans la famille; tandis que Ramatoulaye se montre comme une femme traditionnelle et passive.

Elle ne peut pas tout choisir. La seule décision qu'elle peut prendre, c'est de rester passive : Ramatoulaye, l'héroïne, après vingt-cinq ans de mariage et de bonne relation, est prise pour une seconde épouse parce que la "faute" qu'elle a commise était de tomber soudainement malade.

L'écrivaine critique acerbement la décision subite du mari de prendre une deuxième femme oubliant ainsi toute relation et tout contact conjugal avec la première. Le mari décide subitement de se remarier sans donner le droit à sa première épouse d'exprimer son malaise psychique. Elle n'a qu'à exécuter ses ordres.

Selon Bâ, dès qu'on se rend compte que le nouveau-né est une fille, elle est donc inferieure, esclave, dépendante et soumise finalement à l'homme.

(La situation choquante de la femme...) Dr. Mohamed Abdel Latif 
Mariama Bâ décrit la vie quotidienne des personnages principaux en se concentrant sur Ramatoulaye et Aïssatou pour donner l'occasion aux lecteurs de faire une exploration et même une investigation de la vie intérieure et inconsciente de chaque personnage.

Dans la lettre qu'elle écrit à son amie sénégalaise, Ramatoulaye évoque 1'histoire de deux mariages fondés sur la décision des hommes de prendre une seconde femme comme épouse sans prendre la permission de la première. Elle se rappelle son mariage avec Modou Fall et celui de son amie d'enfance, Aissatou avec Mawdo Bâ.

Ramatoulaye a une cinquantaine d'années et douze enfants avec Modou Fall, mais après vingtcinq ans de mariage et de bonne entente, Modou épouse la jeune fille Binetou, l'amie de leur fille Daba.

Selon la conception de l'écrivaine, la polygamie est considérée comme forme d'isolement et de distanciation humiliante pour les femmes concernées et les sénégalaises en général. Le fait que les

(La situation choquante de la femme...) Dr. Mohamed Abdel Latif 
hommes optent pour la polygamie montre leur incapacité à entretenir des relations fidèles voire égales entre elles.

Quand Aïssatou quitte son mari Mawdo, il prend en considération qu'il a commis une erreur, mais il n'admet pas sa faute ni sa trahison.

Pourtant, il ne change pas de comportement. De plus, Modou ne se repentit pas de tout ce qu'il avait fait contre Ramatoulaye, au contraire, il va plus loin, à savoir la trahir avec sa meilleure amie qui lui rendait visite de temps en temps sans respecter ni ses émotions ni la fidélité conjugale. De plus, il ne respecte ni les mœurs ni les traditions de la société de sa première épouse en ce qui concerne les pensions alimentaires complètement oubliées et négligées après son mariage avec Binetou.

Outre les besoins matériels, il ne donne ni soin ni affection ni à Ramatoulaye ni à leurs enfants ; au contraire, il les laisse mener une vie de vagabondage et abandonne les enfants à l'école buissonnière. Pour assouvir ses désirs charnels, Modou a cherché à trouver une deuxième épouse parce qu'il veut avoir

(La situation choquante de la femme...) Dr. Mohamed Abdel Latif 
une jeune femme qui lui fait plaisir au niveau sexuel.

Mais selon l'héroïne, il faut respecter le vieillissement, la bonne relation conjugale et la force de l'amour entre le couple. Pendant un moment, Ramatoulaye pense le quitter parce qu'elle n'accepte point être une coépouse :

«J'étais offusquée. Il me demandait compréhension. Mais comprendre quoi? La suprématie de l'instinct? Le droit à la trahison ? La justification du désir de changement ? Je ne pouvais être l'alliée des instincts polygamiques. Alors comprendre $q u o i »(B a ̂, 1979$, p. 70)

Pour Mariama Bâ «toutes les femmes n'acceptent jamais la polygamie par cœur de gaieté. Si elles l'acceptent, c'est par contrainte (....) (Dia, 1979, p. 22)

Donc, il est inconstatable que la religion joue un rôle très important dans la société sénégalaise ; c'est ce qu'affirme Miriam Murtuza dans son article qui s'intitule « le mariage, la divorce et la polygamie

(La situation choquante de la femme...) Dr. Mohamed Abdel Latif 
au Sénégal, interaction du genre, de la religion et de la classe sociale dans l'cuvre de Mariama Bâ» (Murtuza, 2004). Elle dit que la plupart des sénégalais sont musulmans, par conséquent, la religion exerce une grande influence dans l'ensemble du pays. À cause de la religion et son rapport avec le mariage, Ramatoulaye est fidèle à Dieu, donc elle restera à jamais attachée à son mari et son foyer conjugal, elle ne peut pas imaginer la vie d'un seul côté. Tout cela a pour objectif d'atteindre la satisfaction de Dieu et gagner enfin le paradis, comme le montre cet extrait «Je suis de celles qui ne peuvent se réaliser et s'épanouir que dans le couple » (Bâ, 1979, p. 89). Autre exemple qui met en évidence l'influence de la religion musulmane sur Ramatoulaye. : Quand Modu est mort, Ramatoulaye choisit le partage de son foyer conjugal avec sa coépouse, Binetou à cause de la société et ses traditions, d'une part, et les rites de l'Islam, d'autre part.

De Beauvoir a aussi son propre point de vue concernant, le mariage, la femme prise comme coépouse, le rapport homme-femme et le thème de (La situation choquante de la femme...) Dr. Mohamed Abdel Latif 
la polygamie. Elle dit que le mariage traditionnel est la cause principale de sa passivité et son angoisse existentielle. Selon de Beauvoir, le mariage est le destin traditionnel de la femme. Il rend la femme passive (...) (Beauvoir, 1949, p. 51)

Autre forme de marginalisation de la femme chez Mariama Bâ, c'est l'inégalité des sexes au sein de la famille sénégalaise influencée par l'Islam. Selon la pensée de Bâ, en recourant à la théorie critique de Louis Tyson (TYSON, 2001, p. 44), la femme dans certaines régions patriarcales $\mathrm{du}$ Sénégal est très marginalisée, elle est 'l'Autre', ses valeurs et ses droits sont indéniables par rapport à l'homme. Cela est dû à la culture, l'éducation sociale et psychologique que reçoivent les Sénégalais dès leur naissance. Même dans l'héritage, l'homme hérite deux fois plus que la femme en s'inspirant de la religion musulmane. L'héritage est donc inégalitaire entre eux.

De plus, en ce qui concerne le pouvoir, Mariama Bâ critique acerbement la discrimination et la distribution du pouvoir inégalitaire dans les affaires

(La situation choquante de la femme...) Dr. Mohamed Abdel Latif 
publiques, la société, et en particulier la politique. Bien que la femme soit comme l'homme, un être indépendant, elle est inégale au niveau de la hiérarchie politique et sociale sous prétexte qu'elle est un sexe faible et que sa nature biologique l'empêche de pratiquer certaines activités. Elle est donc persécutée par l'homme en la forçant à devenir l'Autre, La femme lui est ainsi subordonnée. Dans une telle situation, elle est considérée donc comme handicapée et incapable d'assumer même sa propre responsabilité sans le soutien de l'homme, comme la qualifie Simon De Beauvoir :
"La femme a toujours été, sinon l'esclave de l'homme, du moins sa vassale; les deux sexes ne se sont jamais partagé le monde à égalité ; et aujourd'hui encore, bien que sa condition soit en train d'évoluer, la femme est lourdement handicapée » (Beauvoir, 1949, p. 47).

C'est la même déclaration du personnage dans L'Enfant de sable. Il dit que les rapports sociaux sont régis par la loi de la violence vis-à-vis de la

(La situation choquante de la femme...) Dr. Mohamed Abdel Latif 
femme et que la déchéance physique et psychologique accentue «cette atmosphère », atmosphère oppressive pleine de dégradation, de malaise et de déchirement qui interprète le destin de la femme dans le roman de Ben Jelloun (...) (Andréa, N013 2007). De plus, à cause de la tyrannie qu'elle subit de la part de l'homme, elle subira un vieillissement précoce beaucoup plus que son conjoint.

Les théories de Simone de Beauvoir susdites nous paraissent utiles parce qu'elles luttent contre l'idée des différences biologiques entre les sexes.

Selon la théorie de Beauvoir «on ne naît pas une femme, on le devient »(Jelloun, 1985, p. 23).

Si Modu accuse sa femme Ramatoulaye d'être atteinte de froideur sexuelle, le père dans l'Enfant de sable accuse sa femme d'être incapable de mettre au monde un garçon et qu'elle est responsable des malheurs qui s'abattent sur eux; il s'agit de la culpabilité, comme le montre cet extrait «son époux, toujours mécontent, à la fierté froissée, à l'honneur perdu, la rendait responsable du malheur qui s'était abattu sur eux»

(La situation choquante de la femme...) Dr. Mohamed Abdel Latif 
(Jelloun, 1985, p. 27). Le père poursuit sa critique en faisant une comparaison entre elle et Eve, figure religieuse, qui était la cause principale du péché originel et subséquemment de l'expulsion des humains du paradis. Par conséquent, la mère fut l'objet d'un discours de dépréciation et de déprime reconnaissant qu'elle est la cause du drame humain sur la terre. Donc, elle doit se punir : «Elle se mettait aussi à se désintéresser de ses filles. Elle leur en voulait d'être là, se détestait et se frappait le ventre pour se punir » (Jelloun, 1985, p. 19).

Pour renforcer aussi le sentiment de culpabilité chez son épouse, il s'octroie le titre de médecin en s'appuyant sur une fausse ordonnance scientifique qui prétend que sa femme ne sera jamais capable de lui donner un garçon et qu'elle sera à jamais infertile, puisque nous vivons dans une société patriarcale où la parole de l'homme est sacrée tandis que celle de la femme est négligée, le père n'a qu'à renforcer ses reproches en disant :

«Au bout de ta septième fille, j'ai compris tu portes en toi une infirmité : ton ventre ne peut concevoir d'enfant mâle; il est fait de telle sorte qu'il ne donnera - à perpétuité - que des femelles. Ça

(La situation choquante de la femme...) Dr. Mohamed Abdel Latif 
doit être dî̀ à un manque d'hospitalité qui se manifeste naturellement et à ton insu à chaque fois que la graine que tu portes risque de donner un garçon(...) (Jelloun, 1985, p. 24). Démoralisée, diminuée, condamnée et soumise aux fausses accusations de l'homme, la femme n'a qu'à se cloisonner sur elle-même. La mère représente l'état des milliers de femmes qui sont emprisonnées par leurs maris, elles sont donc condamnées à la solitude et à l'isolement.

Cet emprisonnement n'est qu'une autre forme de la marginalisation de la femme en Afrique soit au Maroc, soit au Sénégal.

\section{II- L'emprisonnement de la femme}

L'emprisonnement du personnage féminin dans le roman francophone, que nous étudions ici, est le reflet des idées archaïques et figées dans des pays où la femme reste «Une éternelle mineure, subordonnée à l'homme et lui devant obéissance !» (Andréa, N013 2007). Pour dire les choses sans exagération, la femme dans l'espace romanesque du roman francophone africain a un statut de prisonnière. Son existence dans la vie est toujours dépendante et soumise à l'analyse du

(La situation choquante de la femme...) Dr. Mohamed Abdel Latif 
mâle. Nous allons essayer de le prouver à travers la pensée de l'écrivain francophone qui nous offre un tableau remarquable d'une société où l'homme et la religion sont tenus au sommet du pouvoir. A l'écart de ces deux pôles, la femme n'a aucune position. Alors, elle n'a qu'à se plier automatiquement à la bonne volonté de l'un et de l'autre. Sa soumission porte la marque de l'enfermement physique d'une part, et affecte par conséquent ses mouvements et ses réactions : «Et, pour toutes ces femmes, la vie était plutôt réduite. J'étais secrètement content de ne pas faire partie de cet univers si limité »(Jelloun, 1985, p. 36). Les synonymes employés ci-dessus comme "réduite' et "limité" renforcent bel et bien le choix lexical de cet isolement qui frappe ces êtres.

En gros, la femme a un statut de prisonnière. Son existence dans l'espace romanesque est soumise à l'autorité du mâle. C'est lui qui organise l'emploi du temps de sa femme; à savoir, il a le droit de s'imposer à ses déplacements. A vrai dire, nous sommes devant dans un monde clos dans lequel elle vit. Les restrictions que le père impose à sa femme

(La situation choquante de la femme...) Dr. Mohamed Abdel Latif 
ainsi qu'à sa famille témoignent de cette autorité patriarcale :

«Ahmed dit ses conditions. Elle ne sortirait de chez elle que pour aller au bain et à l'hôpital »(Jelloun, 1985, p. 71)

Ces sorties sont liées à des infractions si elles ne respectent pas les réglementations qui régissent les conduites féminines : « Le maître de céans établit les règles de la vie en couple de manière unilatérale, lesquelles tendent à immobiliser la femme à longueur de journée dans la maison aux allures d'une réelle geôle. La claustration est la marque déposée de son existence. Elle s'y étiole, s'y consume avant qu'une main sereine et bonne ne vienne la délivrer de cette prison où lentement on l'a enfermée » (Jelloun, 1985, p. 133).

Toutes ces agressions constituent une violation solennelle des chartes humaines et universelles des libertés fondamentales et des droits de l'homme de 1948.

D'autre part, l'enferment physique va de pair avec l'enferment verbal, à savoir, la prise de la parole est toujours consacrée à l'homme, c'est lui qui dirige la discussion, qui monopolise l'entretien, alors que les femmes sont claustrées dans le silence et la répétition de ce que disent leurs maris, elles

(La situation choquante de la femme...) Dr. Mohamed Abdel Latif 
parlent très peu. L'évaluation de la répartition de la parole est toujours en faveur de l'homme, les femmes n'ont qu'à écouter. Même les interventions sont toujours précises et concises.

Donner la parole à un interlocuteur, cela veut dire lui donner le pouvoir d'exister et de se faire entendre et comme a dit Sartre «je parle, donc j'existe » (Sartre, 1964, p. 44)

Le fait de ne pas parler réduit la personne au statut d'esclave alors que l'interlocuteur est son maître :

Dans le roman de Tahar Ben Jelloun, Hadj Ahmed ne fait pas de tergiversations pour exprimer la réclusion de la parole féminine. Le dialogue cidessous révèle brutalement cette réalité :

«- Moi, je n'ai rien décidé.

- C'est vrai ! Dans cette famille, les femmes s'enroulent dans un linceul de silence..., elles obéissent ; toi, tu te tais et moi j'ordonne ! (Jelloun, 1985, p. 54).

L'homme a créé un stratagème de communication où la femme n'a pas le droit ni à la parole ni à donner son avis. Toute énonciation

(La situation choquante de la femme...) Dr. Mohamed Abdel Latif 
commence en fonction de sa personne, c'est à partir de lui et pour lui que le dialogue commence. De plus, c'est seulement le discours du mari qui est légitimé et par conséquent fait autorité ; il fait la norme.

La soumission fidèle de la femme à ce discours se voit dans ces propos de Hadj Ahmed :

'L'enfant que tu mettras au monde sera un mâle, ce sera un homme, il s'appellera Ahmed même si c'est une fille! J'ai tout arrangé, j'ai tout prévu. Toi, bien entendu, tu seras le puits et la tombe de ce secret. Ton Bonheur et même ton nom en dépendront", (Dominique, Novembre-Décembre 2000).

Même le verbe qui constitue le noyau de la phrase est complètement interdit à la femme a cause de l'ordre patriarcal du père comme l'éprouve Dominique le Boucher :’' c'est "une parole tue le plus souvent, interdite, une parole qui noue entre elles des vies ignorées" (Jelloun, 1985, p. 165). Il faut dire aussi que cet acte engendre un sentiment terrible et profond dans le soi. L'épouse n'a qu'à s'enliser dans un silence absolu. De plus, l'homme l'a empêchée involontairement de toute possibilité de parler ou de communiquer avec l'Autre. Si on lui propose quelque chose en vue de parler, elle dit je n'ai pas le

(La situation choquante de la femme...) Dr. Mohamed Abdel Latif 
choix de parler et elle se tait sur le coup, comme le montre la citation suivante :

« - Et toi, Fatouma, tu ne dis rien... Quel est ton point de vue ?...

- Oui, je ne dis rien, parce qu'une femme, dans ce pays, a pris l'habitude de se taire

Quand bien même elle l'énoncerait, cette opinion sera balayée du revers de la main, n'entrera pas en ligne de compte: "J'ai droit à la parole parce que ça n'a pas d'importance » (Jelloun, 1985, p. 166)

Nous observons dans cet extrait que le silence est devenu quelque chose d'ordinaire et par conséquent fait partie de son identité féminine. Même les mots qui sortent de sa bouche sont censurés, le choix du vocabulaire est obligatoire, car la société y exerce une censure permanente. De surcroit, dans le roman, nous constatons que les mots de la femme, qui interprètent ses impulsions sont censurées et contrôlées. Les femmes n'ont jamais le droit d'utiliser des mots qui les fascinent et leur permettent de se libérer de leur défoulement, tels : "sperme"...,"couilles"...,"vagin"...".

(La situation choquante de la femme...) Dr. Mohamed Abdel Latif 
Donc, on perçoit la fille en tant qu'être humain dans la société marocaine qui doit être fine et subtile et qui n'a pas le droit de dire de gros mots .De plus, elle n'a pas le droit de s'émanciper ni d'exprimer ses impulsions, parce que c'est une femme et que toutes les femmes doivent se caractériser par la politesse. Les hommes ne se rendent pas compte que cette action a des effets néfastes sur l'état psychique de la femme.

Selon la théorie de Sigmund Freud, ce blocage linguistique ne fait qu'augmenter son refoulement. Tout est bien considéré, ce recrutement de la parole occupe une fonction idéologique dans l'œuvre romanesque de Ben Jelloun.

L'indifférence de la femme vis-à-vis de la cruauté de l'homme annule toute volonté de révolte ou d'affirmation de soi et si jamais il y a une révolte, elle sera avortée et n'aura aucun effet dans la société. Donc, le silence, qui fait partie inhérente de la femme, n'est autre chose qu'une déclaration de la mort symbolique du personnage féminin. La situation choquante existentielle dans laquelle se

(La situation choquante de la femme...) Dr. Mohamed Abdel Latif 
trouvent les personnages féminins du roman fait l'objet de l'intérêt dont nous nous proposons à présent d'étudier quelques aspects tels: le conservatisme et la tradition.

D'autre part, Ben Jelloun a pris pour source d'inspiration le titre $\mathrm{du}$ roman épistolaire qui s'intitule Une si longue lettre pour lancer l'image d'une femme africaine prise dans une situation dangereuse, il s'agit du conservatisme traditionnel. Selon Bernard Mbassi, les personnages féminins, soit au Maroc, soit au Sénégal, autour duquel se focalise notre étude, dressent le portrait d'une société fortement «masculin pluriel» (Bernard, Janvier 2004). Il dit que dans le monde francophone, surtout en Afrique, l'homme et la religion ont associé leurs efforts afin de réduire l'espace existentiel de la personnalité de la femme et d'en faire une créature asservie.

La réclusion de la femme au Maroc était la même au Sénégal sous la plume de Mariama Bâ. Elle critique aussi la distinction entre hommes et femmes à cause de leur différence biologique. Selon

(La situation choquante de la femme...) Dr. Mohamed Abdel Latif 
la conception de Bâ, toutes les femmes qui sont nées en Afrique, souffrent d'une grande isolation au sein de la société sénégalaise, la femme sénégalaise trouve une grande complexité à s'affirmer devant toutes les couches sociales de ce pays. L'écrivaine dit dans son roman épistolaire intitulé Une si longue lettre qu'il n'y a pas de différence entre une femme et une négresse, les deux sont égales comme le mentionne Angelfors : «Dans l'auvre de Ba, on trouve une préoccupation constante de la notion de "différence" entre hommes et femmes. Tous les personnages féminins d'origine africaine de Bâ se débattent avec cette différence biologique » (ANgelfors, no 77,2009)

Si Benjelloun montre la femme passive et incapable d'avoir l'auto-affirmation en ce qui concerne son identité et sa vie personnelle, les personnages de $\boldsymbol{B} \hat{\boldsymbol{a}}$ refusent cet emprisonnement et réclament la révolte contre cette oppression et cette discrimination de genre. Prenons par exemple, Aissatou qui refuse le rôle secondaire de la femme que lui attribue son mari, et par conséquent elle le quitte. Elle décide de divorcer et de continuer sa vie à l'étranger notamment aux Etats unis en

(La situation choquante de la femme...) Dr. Mohamed Abdel Latif 
accomplissant ses projets sans que ce divorce soit un obstacle pour elle.

Ici la marginalisation se voit dans la négligence, lorsqu'on devient pauvre. Aissatou était au début du mariage riche lorsqu'elle a épousé son mari. Au fil du temps, elle devient pauvre, parce que c'est elle qui assumait les dépenses de son foyer. La mère de Mawdo, qui est la cause de leur séparation, convainc son fils de se marier avec une bijoutière pare qu'elle est plus riche que sa première femme et que selon la mère, c'est l'argent qui donne plus de valeur à la femme. Dès que Mawdo a pris une seconde épouse, Aissatou quitte la maison en écrivant cette lettre à son mari :

"Des lors, tu dégringoles de l'échelon supérieur, de la responsabilité où je t'ai toujours hisse. Je me dépouille de ton amour, de ton nom. Vêtue du seul habit valable de la dignité, je poursuis ma route. Adieu, Aissatou » (Bâ, 1979, p. 45)

Pour le cas de Ramatoulaye, c'est tout à fait différent, à savoir que c'est Aissatou qui a refusé d'être la deuxième femme pour mari, Ramatoulaye

(La situation choquante de la femme...) Dr. Mohamed Abdel Latif 
n'accepte pas de vivre hors du foyer conjugal. Pour elle, une vie en dehors du mariage est impossible.

En fin, Modou contraint Ramatoulaye à vivre seule en prenant soin de ses enfants. Abandonnée et elle ne trouve pas de quoi manger et est obligée de travailler pour gagner sa vie afin d'assurer la nourriture et la bonne sécurité pour elle ainsi que pour ses enfants. Donc, nous pouvons dégager que l'identité de la femme Africaine est limitée dans la maternité, l'élévation des enfants et les tâches ménagères.

D'autre part, les hommes en Afrique sont indignés lorsque leurs femmes mettent au monde un nouveauné de sexe féminin. Les épouses confisquent alors leur genre, cela ne vient que par la voie de masculinisation de leur genre, ce déni identitaire et sexuel sera l'objet de notre analyse suivante :

\section{La dénégation identitaire et sexuelle de la femme francophone}

L'effacement du genre féminin était l'un des mauvais fléaux duquel souffrait la femme depuis l'aube du temps jusqu'au tournant de l'année deux

(La situation choquante de la femme...) Dr. Mohamed Abdel Latif 
mille. Les deux romanciers ont décrit ce drame féminin à travers les personnages de leur œuvre romanesque. Ils analysent le drame du genre féminin qui est dénué de son nom et de sa singularité féminine, tout simplement à cause de son sexe. L'Enfant de sable en est un exemple, ce roman concrétise l'effacement du genre féminin. Alors que Hadj Ahmed désire impatiemment un garçon d'une huitième naissance, la volonté de Dieu lui donne un nouveau-né de sexe féminin. L'épouse dès qu'elle découvre que le bébé est une fille se met à pleurer, et le père sur le coup, afin d'éviter un tel scandale dans son entourage, selon lui, dit à la sage femme que la fille aura le prénom d'un garçon par mensonge. Ce déni identitaire et sexuel est marqué grammaticalement par l'emploi du masculin à la place du féminin comme le montre cet extrait « Regarde, regarde bien, c'est un garçon ! Tu viens après quinze ans de mariage de me donner un enfant, c'est un garçon, c'est mon premier enfant, regarde comme il est beau » (Jelloun, 1985, p. 28)

C'est ainsi que la fille sera un garçon au corps d'une femme en la dépossédant de son identité et de sa

(La situation choquante de la femme...) Dr. Mohamed Abdel Latif 
nature biologique en la tenant en secret. La substitution du masculin au féminin montre à quel point la valeur de la fille est altérée, bafouée et dépouillée dans cette société, « $\boldsymbol{L} \boldsymbol{a}$ distribution masculin/féminin à laquelle nous assistons dans l'aeuvre dénie dans une certaine mesure toute existence individuelle à la femme » (Mokhtar, $\mathrm{n}^{\circ}$ 10-2008)

Dans le même ordre d'idées, tout le monde sait que le prénom est le premier moyen qui qualifie un personnage et qui assure son existence aux yeux des autres, mais en Afrique ou dans la société arabe, c'est autre chose; à savoir, il est impossible d'appeler les femmes par leurs propres prénoms. C'est la raison pour laquelle le prénom est considéré comme l'un des thèmes les plus importants dans l'Enfant de sable.

Dans ce roman, Ben Jelloun, montre que la désignation et la dénégation des femmes de leurs noms sont une grande problématique. Prenons par exemple l'épouse d'Ahmed qui est singularisée par son nom de famille. Même les filles, elles ne sont même pas individualisées par leurs prénoms, « Toutes sont réduites soit à une personne (La situation choquante de la femme...) Dr. Mohamed Abdel Latif 
pronominale, soit à un terme générique. Morceaux choisis : "sa femme" " (Jelloun, 1985, p. 19), sans compter d'autres qualifications comme ", la femme, ", la pauvre femme', ...etc.

En gros, Les femmes de cette famille sont tombées dans l'anonymat. Elles sont tout simplement dénuées de leur particularité, figées dans des appellations stéréotypées qui ne font qu'amplifier le tragique de leur condition et existence. Il ressort de ces exemples que cette forme de «désignation d'autrui a pour objectif de requérir de la part de celui qui nomme une prise de position à l'égard de ce qui est nommé. » (Siblot, 1997).

Pour conclure, nous pouvons constater que cette identité truquée et tronquée par l'homme montre qu'il se positionne comme l'unique dépositaire du sens. Elle donne à lire et à voir le mal-être des femmes écrasées et qui ne "s'appartiennent pas mais sont définies par rapport à un homme(...) (Cécile, no13, Juin-Juillet 2010).

D'une autre manière, la dénégation identitaire réside dans la peau noire de la femme. À titre d'exemple, Sorraya, l'amie de Ramatoulaye, lors de son séjour

(La situation choquante de la femme...) Dr. Mohamed Abdel Latif 
en Franc se plaint de sa dénégation identitaire à cause de sa peau noire en disant :

«J'ai toujours appartenu à une minorité, reprit-elle. Vous ne m'acceptiez pas parce que j'estimais que j'avais certains droits, que tout n'était pas bon dans nos traditions. En France, jamais je ne serai considérée comme une blanche.je n'appartiens à rien, simplement parce que je suis nègre(...) (Bâ, 1979, p. 89)

De surcroit, Ramatoulaye, après avoir divorcé, a refusé la demande de son cousin Daouda Dieng qui l'aime depuis sa jeunesse parce qu'il la considère comme un objet sexuel fait pour satisfaire ses besoins sexuels. Elle préfère être toute seule plutôt que de se marier avec un homme qu'elle n'aime pas et qui voit en lui la virilité absolue : «Tu oublies que j'ai un cour, une raison, que je ne suis pas un objet que l'on se passe de main en main. Tu ignores ce que se marier signifie pour moi : c'est un acte de foi et d'amour, un don total de soi à l'être que l'on a choisi et qui vous a choisi " (Bâ, 1979, p. 88)

(La situation choquante de la femme...) Dr. Mohamed Abdel Latif 


\section{Conclusion}

$\mathrm{Au}$ terme de notre recherche, nous avons constaté que les femmes sont victimes des habitudes et des traditions qui sont encore figées dans la société francophone, soit au Maroc, soit au Sénégal et qu'elles ont un effet très négatif sur la position de la femme dans la société. De surcroit, les deux écrivains, à travers leur œuvre romanesque, ont su bien décrire l'assujettissement de la femme au pouvoir de l'homme qui exerce un pouvoir absolu sur elle. L'enfant de sable, est un bon exemple du roman sociologique qui décrit les difficultés d'une société à rendre la femme au même pied que l'homme. Selon la pensée de Tahar Ben Jelloun, elle est opprimée dans les pays arabes en général et au Maroc en particulier. Et il a réussi à bien analyser cette situation avec beaucoup de justesse et d'objectivité comme le mentionne cet extrait :

"Avec l'art, la littérature est sans doute le meilleur moyen pour traduire cette expérience en langage et la mettre en forme." (Alia, le 26 juin 2005)

(La situation choquante de la femme...) Dr. Mohamed Abdel Latif 
Cela a été prouvé d'après l'histoire personnelle de l'épouse et des filles de Hadj Ahmed. De l'autre côté, Mariama Bâ, prend comme source d'inspiration ses expériences personnelles de genre pour nous relater les problèmes auxquels les femmes sont confrontées ; il s'agit de problèmes de discrimination, d'oppression sociale et de misogynie. Nous avons prouvé cela à travers Une si longue lettre, une œuvre romanesque autobiographique où la romancière se sert de son écriture au féminin pour concrétiser l'histoire des femmes africaines qui luttent de façons variées contre l'injustice et le patriarcat de l'homme auquel elles se trouvent soumises. Elles sont conscientes de leurs problèmes d'inégalité et de discrimination, c'est pour cela qu'elles ont pris des mesures concrètes pour se libérer du joug de patriarcat. Il nous reste à dire que, grâce aux écrits au féminin de Mariama Bâ surtout son chef-d'œuvre Une si longue lettre, la condition des femmes s'améliore. En gros, la situation de la femme dans le roman francophone fait du progrès et pourtant elle est toujours considérée comme l'Autre.

(La situation choquante de la femme...) Dr. Mohamed Abdel Latif 
Enfin, s'il y a une ressemblance chez les deux écrivains en ce qui concerne la description de la femme dans le roman francophone, l'image de la femme africaine en est la meilleure par rapport à la femme marocaine qui vit toujours en plein asservissement malgré les écrits de Ben Jelloun.

(La situation choquante de la femme...) Dr. Mohamed Abdel Latif 


\section{Bibliographie}

Alia, T. (le 26 juin 2005). Alia, Tabaï, "Extrait d'un texte lu au Congrès mondial du Conseil International d'Etudes Francophones à Sinai en Roumanie, sur le thème de "Cris et chuchotements dans la littérature contemporaine". le congrès mondial du Conseil International d'Etudes Francophones (pp. Alia, Tabaï)

Andréa, C. (N013 2007, ,Juin-Juillet). Du drame individuel à la tragédie collective. Interculturel francophone, 88.

ANgelfors, C. ( no 77,2009). ANgelfors, Christina : "Féminitude et Négritude: discours de genre Féminitude et Négritude:discours de genre et discours culturel dans l'œuvre de Mariama Bâ ». Paris: ANgelfors, Christina : «Féminitude et Négritude : discours de genrePrésence francophone.

Assad, C. (2012). La femme entre tradition et modernité dans le roman francophone. Paris: Seuil.

ASSARAF, A. (1993). Quand dire,c'est lier,les Nouveaux actes sémiotiques. les Nouveux Actes sémiotiaques, 75.

Bâ, M. ( 1979). Une si longue lettre. Paris: Nouvelles Éditions Africaines.

Béatrice, R. G. (1997). I'oeuvre romanesque de Mariama Ba. Paris: I'Harmattan.

Beauvoir, S. D. (1949). Le Deuxième sexe II, Éditions Gallimard, .p51. Paris: Éditions Gallimard,Idées.

Bernard, M. (Janvier 2004). "Structure et apories de l'argumentation féministe dans une si longue lettre de Mariama Bâ ". Revue Langues et Littérature, p.115.

(La situation choquante de la femme...) Dr. Mohamed Abdel Latif 
Cécile, D.-E. ( no13, Juin-Juillet 2010). , "Le personnage androgyne chez Mongo Beti. Interculturel Francophonies, p.100.

Combe, D. (1995). Poétiques francophones. Paris: Hachette.

Dia, A. T. (1979). succès littéraire de Mariama Ba pour son livre Une si longue lettre. Brazzaville: Amina.

Dominique, I. B. (Novembre-Décembre 2000). " Cette fille-là. Lecture/Dialogue" . Littérature/Action, p.142.

Jelloun, T. B. (1985). I'enfant de sable. Paris: Seuil.

Mokhtar, H. ( $n^{\circ} 10-2008$ ). Le personnage féminin : glorification ou anonymat dans La nuit sacrée de Tahar Ben Jelloun. Synergies Algérie, p196.

Mortimer, M. (1988). Entretien avec Assia Djebâr,écrivain algérien,recherche en litterature africaine. Paris: Université Presse.

Murtuza, M. ,. (2004). Le mariage,le divorce et la polygamie au Sénégal,féminisme et post-colonialisme. magazine le monde africain.

Rey, A. (2006). le Robert micro. Paris: Nouvelle édition.

Sartre, J. P. (1964). l'Existentialisme est un humanisme. Paris: Ngle.

Siblot, P. (1997). " De I'un à l'autre : Dialectique et dialogisme de la nomination identitaire in l'autre en discours. Montpellier: Presses universitaires de Montpellier III.

TYSON, L. (2001). Critical Theory Today. London: Garland Publishing.

(La situation choquante de la femme...) Dr. Mohamed Abdel Latif 


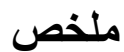

الوضع الصادم للمر اة في روايتين فر انكوفونتين عند طاهر بن جلون ومريما با يتناول هذا البحث بالدر اسه قضية مهمة من قضايا النسوية،تتصل بصورتها في المجتمع الذكوري، وذللك من خلال روايتي"طفل الرمال" للروائي المغربي طاهر بن جلون و "خطاب طويل جدا" للروائية السنغالية مارياما با.وقد توققت الدراسة عند ما يبدو في الروايتين من تناول معايير لوضعية المراة في المجتمع،اذ يبدو في الرواية الاولي تهميش المراة في المجتمع وتعدد محو خصوصيتها في المجتمع، والخجل من كونها انتي، بينما نظهر المراة في رولية"خطاب طويل جدا" علي النقيض تمام. الكلمات المفتاحية: النسوية_ طاهر بن جلون-مارياما با 